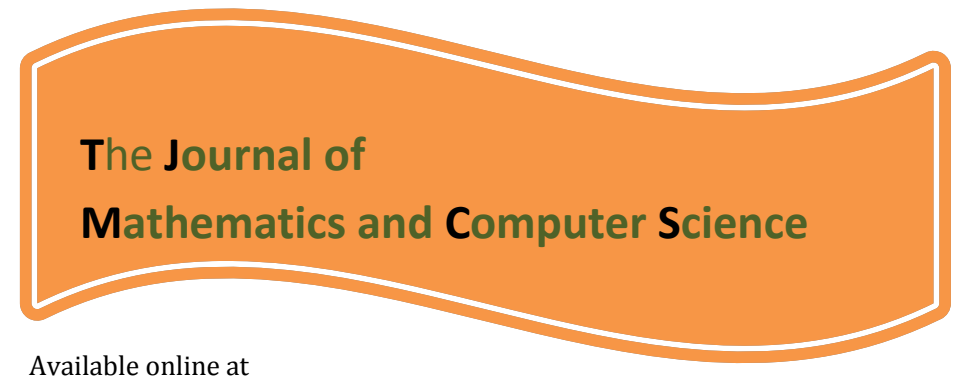

http://www.TIMCS.com

The Journal of Mathematics and Computer Science Vol. 4 No.3 (2012) 428- 435

\title{
Using a Hybrid Simulated Annealing and Genetic Algorithms for Non Fixed Destination Multi-depot Multiple Traveling Salesmen Problem with Time Window and Waiting Penalty
}

\author{
Mohammad Taher Shirafkan ${ }^{*}, 1$, H. Seidgar ${ }^{2}$, J. Rezaian-zeidi $^{3}$, N. Javadian ${ }^{4}$ \\ ${ }^{1,2,3,4}$ Department of industrial engineering, Mazandaran University of Science and \\ Technology, Babol, Iran \\ 'taher_shirafkan@yahoo.com
}

Received: February 2012, Revised: May 2012

Online Publication: July 2012

\begin{abstract}
The non-fixed destination multi-depot multiple traveling salesmen problem (MmTSP) is a generalization of well-known MTSP with several salesmen in each depot. In this research, time window is defined for each depot (city).the salesmen only can service the customers within these time windows and also some penalties are considered for any deviation of start time. The objective function of problem is to minimize the total costs and penalties of the tours. This problem is of a great complexity and belongs to NP-complete class of problems. So the exact algorithms cannot perform the best solution in problems with big dimension. So Meta heuristics algorithm is used to solve these problems efficiently. In this research we used hybrid simulated annealing and genetic algorithms.
\end{abstract}

Keywords: Multiple traveling salesmen, Genetic algorithm, simulated annealing, Time window

\section{Introduction}

Nowadays, with the development of global manufacturing and intense competition among firms and companies, as well as shorter product life cycles, the time required for the different needs of clients in different areas of marketing and different distances of manufacturing companies in distribution of those commodities raw materials and

\footnotetext{
${ }^{*}$ Corresponding author
} 
transportation have made the systems to improve their performance and productivity. Transportation and distribution systems must be able to distribute the product with the lowest cost and in the shortest possible time so that the products are timely delivered to the clients. One of the earliest methods of innovative problem solving " $m$ " path "TSP" the limits set by Russell [1] was introduced. Another innovative approach is based on an exchange of practices for "mTSP" also by Potoyn et al [2] resented. Approach to solve parallel processing mTSP using evolutionary programming first was introduced by Fogel [3]. Wacholder et al [4] developed ANN model Hopfyld - tank for mTSP, but these models is unable to guarantee good answers evaluated too complex. H. Su et al [6] offered a neural network approach to solve mTSP Based on solving " $m$ " standard "TSP" problem. The authors noted that the results are better than that Wacholder et al [4] Mention had been made. Modares et al recently [7] And the Somhom et al [5] introduced selforganizer NN approach for a mTSP minimal-maximal goal function, to minimize the maximum path cost among all the salesmen. The use of genetic algorithms (GA) was introduced first to solve mTSP by Zhang et al [8] Yu et al [9] also, used genetic algorithms to solve mTSP for the design problem. .Rodrigo [10] In 2010, provided an innovative approach to TSPTW that at first step an initial solution is obtained by VNS method,, and next step by using an innovative enhancements algorithm it get the answers better.

\section{Defining the problem}

The non-fixed destination multi depot multiple traveling salesmen problem (MmTSP) is a generalization of well-known mTSP in which more than one salesmen depart from several starting cities (depots) and returns to the one of starting city (depot) that the number of salemens in each depot remain the same at the end as it was in the beginning, form tours so that each city is visited with exactly one salesman. The problem considered in this study to determine the specific number for each salesman is not required to return the seller so that the course proximal have begun on depot route but salesmen that start with the depot course must be equal with that venders who end the course. The research hypotheses are:

- All salesmen are the same.

- Distance between cities (towns and depots) is pre-determined and fixed.

- Of depot (where the destinations are also) is pre-determined and fixed.

- The number of salesmen in each depot is fixed and known in advance.

- Emitted from a depot with a number of salesmen, the salesmen are just starting to come back. Every seller should have at least two cities (other than home) visit and then return to one of the depot. So at least two cities in one way is required.

- Maximum number of cities in a certain direction is measurable.

- Cities time windows and depots are predetermined and fixed.

- The time windows cover the entire windows of cities. 


\section{Genetic Algorithms}

Genetic algorithm was presented in 1975 by John Holland. This is a guided random search algorithm, a technique based on the theory of human evolution. Genetic algorithms are stochastic search techniques based on the normal mechanism and genetic rules of the composition and mutation. Genetic algorithms start with an initial set of random solutions called the initial population. Every member of the population is called a chromosome represents a solution to the problem. Chromosomes evolve during the frequent course of any period that is called a generation. Population may change at any time and create a new generation of near optimal solution, which is stronger than the previous population.

\section{4 .Simulated annealing Algorithm}

A vast space of optimization problems due to the combination of high time computation, use of SA is effective. The main concepts of this algorithm derived from the laws of physics and thermodynamics of melting. Thus, the temperature of the solid, T, due to the increased heat until it becomes liquid. The body temperature gradually decreased. If the decrease of body temperature occurs slowly, the formation of body atom in energy are at its lowest levels. From the perspective of metallurgical engineering the process is trying to put the atoms of the body near together to place the physical state of the body in the best possible position. The relation between physical concepts with hybrid optimization is equal to the combination of different physical situations. Thus the cost of every solution in hybrid optimization problems is synonym with different levels of "E" energy.

\section{The proposed algorithm}

The algorithm is based on an innovative approach to nearest neighbor search (NNS), is used to generate the initial response. This case serves as a heuristic algorithm, first for each path, starting a home that has been allocated is randomly selected. Then it assigns a cost to the city, As long as a city is assigned to the initial depot. The closest neighboring city that is not allocated is continually searching until all cities are assigned to routes. At the end of each route, the closest destination terminals are assigned to each route. In the proposed algorithm, several classes of neighborhoods transfer have been used for this solution. These transfers mechanism are produced based on a two-Replacement, But with a combination of slot allocation vector, the vector switching, 2-opt, and swap between the two ends of the track and not on the same path. In this study, in one direction, it has been used from local improvement algorithm, as a local search, to improve the current solution. These transmission operators are described below. We can assume two different random path of an answer as follows:

Path k: $\left(-d_{1}, \ldots, i-1, i, i+1, \ldots,-d_{2}\right)$

Path 1: $\left(-d_{3}, \ldots, j-1, j, j+1, \ldots,-d_{4}\right)$ 
a - Re-investment operator: for city $\mathrm{i}$, a member of the $\mathrm{k}$, and city $\mathrm{j}$, a member of the 1 , $\mathrm{i}$ will delete the track $\mathrm{k}$, and $\mathrm{l}$ is the path to $\mathrm{j}$. New directions $\mathrm{k}$ and $\mathrm{l}$ are, respectively: $\left(-d_{1}, \ldots, i-1, i+1, \ldots,-d_{2}\right)$

$\left(-d_{3}, \ldots, j, i, j+1, \ldots,-d_{4}\right)$

$\mathrm{b}$ - Switch function: cities $\mathrm{i}$ and $\mathrm{j}$, are interchanging from paths $\mathrm{k}$ and $\mathrm{l}$, respectively. New directions of $\mathrm{k}$ and $\mathrm{l}$, respectively, are:

$$
\begin{aligned}
& \left(-d_{1}, \ldots, i-1, j, i+1, \ldots,-d_{2}\right) \\
& \left(-d_{3}, \ldots, j-1, i, j+1, \ldots,-d_{4}\right)
\end{aligned}
$$

$\mathrm{c}$ - Multi-function switch: for city $\mathrm{i}$ and $\mathrm{i}+1$, a member of the city $\mathrm{j}$ and $\mathrm{k} 1+\mathrm{j}$, a member of the 1 , new routes will include:

$$
\begin{aligned}
& \left(-d_{1}, \ldots, i, i+1, j+2, \ldots,-d_{2}\right) \\
& \left(-d_{3}, \ldots, j, j+1, i+2, \ldots,-d_{4}\right)
\end{aligned}
$$

d- Operator switching depot (or destination): The operator can switch between the two depots beginning or end.

It should be noted that the choice of route for the new neighborhood, only neighbors are concerned that the time window can also move.

\section{Hybrid simulated annealing and genetic algorithm}

Hybrid algorithm implemented in this sector, is similar to simulated annealing algorithm, with the difference that, to obtain a better value of the objective function, the GA algorithm is used. At first, the track of each saleman, generated by the SA algorithm, and based on the located time window in direction of each city, by genetic algorithm, the best time to begin service to every customer is calculated by a merchant in that visits the city.

\section{6-1.Chromosome representation}

In order of chromosome display is to provide a possible solution to the problem. Figure 1, a new design for non-Fixed MmTSP chromosome with 17 cities, 3 salesperson, 2 depot and 2 sellers in the first depot and for the second depot, shows one saleman. This method involves the use of a single chromosome with length of $n+2 m$. Each chromosome shows the way of each saleman. 


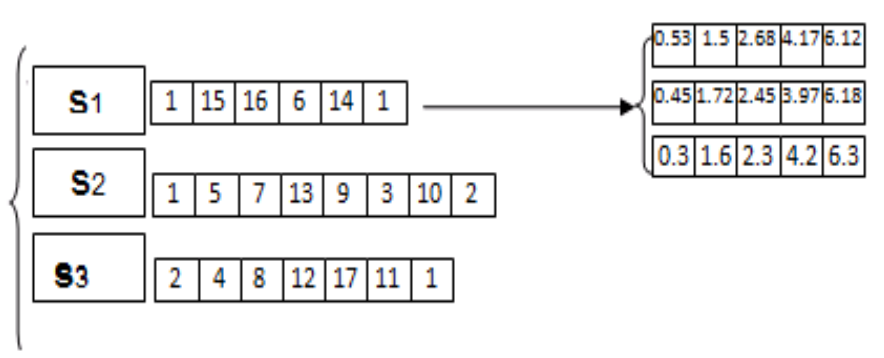

Fig.1. Typical chromosome

In Figure1, the first saleman goes out from depot, and visit cities 15, 16, 6, 14, (with exactly this formation) and returns to the depot one. After finding directions to the dealer (that came out by the SA algorithm) for each route, we use an initial population of possible solutions to the NNS algorithm and implement GA algorithm on it.

\section{6-2.Fitness function}

Fitness function is a criterion for test the quality of chromosomes or the obtained answer. So fitness is the total distance traveled per person and fines by salesmen, is respectively, and based on appearance in the chromosomes.

\section{6-3.Selection of parents}

After a generation produce, it should be selected a number of parents for the next generation of chromosomes in the current generation. These strategies are based on the fitness or chromosomes objective function values.

This study compares the results produced by three conventional methods; the best method was selected, and used in genetic algorithms. These strategies are roulette wheel selection, competitive binary choice, and elitist. Our selected strategy is competitive binary choice.

\section{6-4.Genetic operators}

After selecting the parents, we must apply the appropriate operations on them to produce offspring. It should be noted that in some cases, some of the new produced generations come in to non-production, that to avoid choosing them as a parent for the next generations, a large number, as a fine of non-production is added to the objective function.

\section{6-4-1.Crossover operator}

In this paper, the crossover operator works like this: First, from the population produced for seller's way, as a parent of two chromosomes is selected, then a random number between 1 and $n$ (the length of the chromosome) can be selected. (i.e. gene i) The first child of the first $i$ gene of the parent 1 , and last $n-i$ gene of parent 2 and the second child of the first $\mathrm{i}$ gene of parent 2, and last n-i gene of parent 1 is built. Figures 2 and 3, show the intersection operator. 


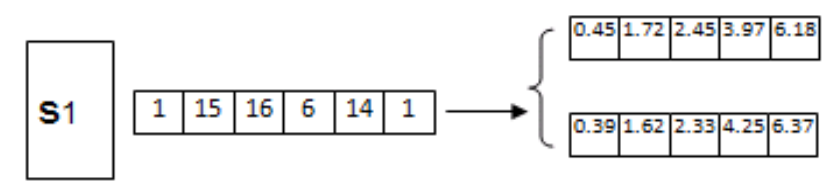

Fig.2. chromosomes before Intersection operator

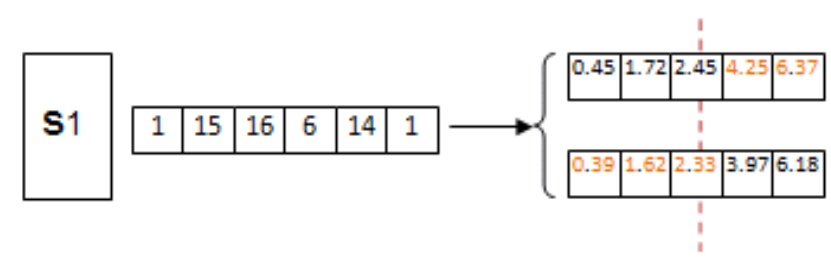

Fig.3. chromosomes after Intersection operator

\section{6-4-2.Mutation operator}

Two types of mutation operator are designed for the problem that is shown in figure 4, and 5 . In the first method, a chromosome is selected randomly from the population as a parent, and all its Ti-ei chromosomes is calculated ( $\mathrm{Ti}$, beginning service in city $\mathrm{i}$, and ei is opening of time window in city i.) Then all Ti decreases from minimum of Ti-ei. In this case at least the beginning of one of the genes reaches to ei, and other genes begin sooner by minimum size of Ti-ei. The second type of mutation operator acts that all genes beginning times, take to the time ei. (Assuming first chromosome in initial population of chromosomes is selected for the specified path)

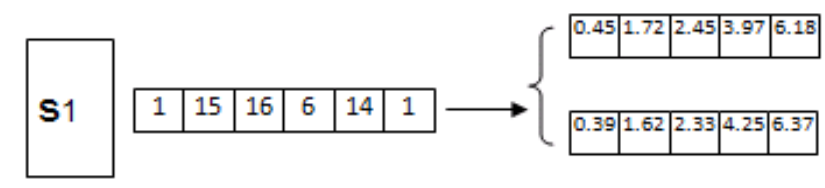

Fig.4. Chromosome before mutation operator

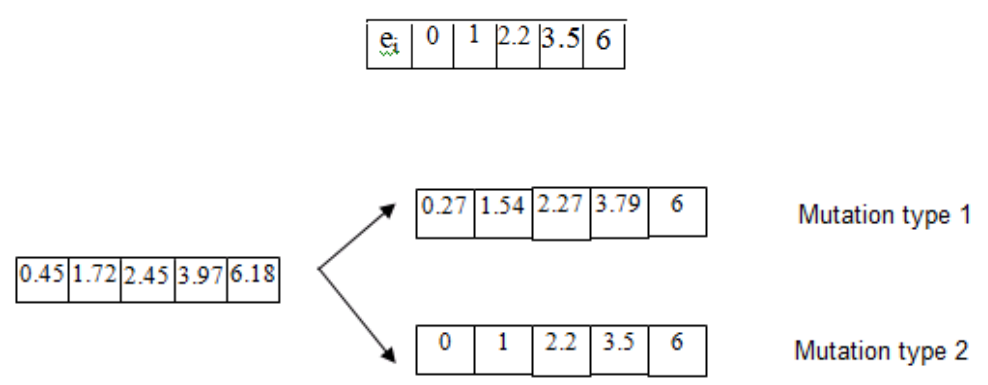

Fig.5. Chromosome after mutation operator for type 1 and 2

\section{6-5.Stopping criterion}


The measure is intended to stop the algorithm, is the maximum number of generations.

\section{Computational Results}

In order to evaluate the performance of the proposed algorithm in Table (1) several problem examples designed and are solved by the proposed algorithm, the data follow the uniform distribution. The cost of having a uniform distribution [1.10], with a fine of uniform distribution [1.10], the service time has a uniform distribution $[0,1]$.

\begin{tabular}{|c|c|c|c|c|c|}
\hline \multirow[t]{2}{*}{ index } & \multirow[t]{2}{*}{$\begin{array}{l}\text { number of } \\
\text { cities(n) }\end{array}$} & \multirow[t]{2}{*}{$\begin{array}{l}\text { number of } \\
\text { salesmen }(m)\end{array}$} & \multirow[t]{2}{*}{$\begin{array}{l}\text { number of } \\
\text { depots(d) }\end{array}$} & \multicolumn{2}{|c|}{$\begin{array}{l}\text { number of salesmen } \\
\text { in each depot(mk) }\end{array}$} \\
\hline & & & & $\mathrm{m} 1$ & $\mathrm{~m} 2$ \\
\hline M1 & 6 & 2 & 2 & 1 & 1 \\
\hline M2 & 7 & 2 & 2 & 1 & 1 \\
\hline M3 & 8 & 2 & 2 & 1 & 1 \\
\hline M4 & 9 & 4 & 2 & 2 & 2 \\
\hline M5 & 10 & 4 & 2 & 2 & 2 \\
\hline M6 & 10 & 4 & 2 & 3 & 1 \\
\hline
\end{tabular}

Table1. Typical problems generated

The point that must be noted is that a bad set of parameters for each algorithm can be obtained low-quality answers. As a result with using sensitivity analysis, the proposed algorithm parameter values are given. Table (2) the value of the objective function, the exact value of the standard deviation (Lingo), and solution time with the proposed algorithm is measured in seconds. Each problem ran 20 times and its average has been considered.

$G A P \%=\frac{O V_{(S A-G A)}-O V_{L I N G O}}{O V_{L I N G O}} \times 100$

Above results indicate that the proposed algorithm is efficient, accurate methods for solving the problem by increasing the size of the time, is on the rise. But the proposed algorithm with increasing the size of problem, reaches good answer in the shortest possible time. (An answer which has a little distance of the optimal solution), it implies that the algorithm is efficient.

\begin{tabular}{|c|c|c|c|c|c|}
\hline PROBLEM & \multicolumn{2}{|c|}{ (Lingo) } & \multicolumn{2}{c|}{ (SA_GA) } & \multirow{2}{*}{ GAP\% } \\
\hline \multirow{2}{*}{ M1 } & ov & Time(second) & Ov & Time(second) & \\
\cline { 2 - 5 } M2 & 25.9 & 16 & 26.56 & 195 & 2.5 \\
M3 & 47.15 & 20 & 48.59 & 293 & 3 \\
M4 & 47.9 & 28 & 50.71 & 319 & 5.8 \\
M5 & 65.25 & 33 & 68.08 & 352 & 4.33 \\
M6 & 56.4 & 636 & 60.9 & 426 & 7.9 \\
& 54.6 & 1337 & 57.99 & 408 & 6.2 \\
\hline
\end{tabular}

Table2. Results of the algorithm 


\section{Conclusions}

In this paper, using refrigerating simulations hybrid algorithm, and genetic algorithms, we proposed a suitable solution with the lowest error and time for a traveling salesman problem with multiple depots of non-fixed, and with considering the limitation of time window and fine.

\section{References}

[1]. Russell RA. (1977), "An effective heuristic for the m-tour traveling salesman problem with some side conditions", Operations Research, 25(3), 517-524.

[2]. Potvin J, Lapalme G, Rousseau J. (1989), “A generalized k-opt exchange procedure for the MTSP". INFOR, 27(4), 474-481.

[3]. Fogel DB. (1990), "A parallel processing approach to a multiple traveling salesman problem using evolutionary programming", In: Proceedings of the fourth annual symposium on parallel processing. Fullerton, CA, p, 318-326.

[4]. Wacholder E, Han J, Mann RC. (1989), "A neural network algorithm for the multiple traveling salesmen problem". Biology in Cybernetics, 61, 11-19.

[5]. Somhom S, Modares A, Enkawa T. (1999), "Competition-based neural network for the multiple traveling salesmen problem with minmax objective", Computers and Operations Research, 26(4), 395-407.

[6]. Hsu C, Tsai M, Chen W. (1991), "A study of feature-mapped approach to the multiple travelling salesmen problem", IEEE International Symposium on Circuits and Systems, 3, 1589-1592.

[7]. Modares A, Somhom S, Enkawa T. (1999), “A self-organizing neural network approach for multiple traveling salesman and vehicle routing problems". International Transactions in Operational Research, 6, 591-606.

[8]. Zhang T, GruverWA, Smith MH. (1999), "Team scheduling by genetic search", Proceedings of the second international conference on intelligent processing and manufacturing of materials, vol. 2, p. 839-844.

[9].Yu Z, Jinhai L, Guochang G, Rubo Z, Haiyan Y. (2002) "An implementation of evolutionary computation for path planning of cooperative mobile robots", Proceedings of the fourth world congress on intelligent control and automation, vol. 3.

[10]. Rodrigo Ferreira da Silva(2010). A General VNS heuristic for the traveling salesman problem with time windows, Discrete Optimization 